\title{
Governo municipal, lixo e saúde no contexto Amazônico: Até que ponto as capacidades afetam a gestão dos serviços básicos?
}

\author{
Municipal government, waste and health in the Amazon context: To what extent do capacities \\ affect the management of basic services? \\ Gobierno municipal, residuos y salud en el contexto amazónico: ¿En qué medida las capacidades \\ afectan la gestión de los servicios básicos?
}

\author{
Hortência Araújo de Sousa \\ ORCID: https://orcid.org/0000-0002-5976-1061 \\ Universidade Federal do Oeste do Pará, Brasil \\ E-mail: hortencia.araujo2011@hotmail.com \\ Marco Aurélio dos Santos \\ ORCID: https://orcid.org/0000-0003-3039-6264 \\ Universidade Federal do Oeste do Pará, Brasil \\ E-mail: marcooliveira_santos@yahoo.com.br \\ Léo César Parente de Almeida \\ ORCID: https://orcid.org/0000-0003-1535-5325 \\ Universidade Federal do Oeste do Pará, Brasil \\ E-mail: leocesarparente@gmail.com
}

\begin{abstract}
Resumo
Os cuidados médicos podem prolongar a vida, mas a saúde depende das condições que causam doenças e os resíduos sólidos e o lixo são fontes de diversos problemas de saúde. A ausência de uma postura mais ativa do Estado pode ampliar os problemas de saúde oriundos da má gestão dos resíduos sólidos e do lixo. Trabalhos têm indicado problemas na gestão do lixo em uma cidade do contexto amazônico. Nesse sentido, o objetivo deste artigo é identificar quais as causas dos problemas da má gestão dos resíduos sólidos e do lixo nesta cidade no contexto amazônico a partir da análise destes trabalhos. Para tanto, recorreu-se à análise documental de um conjunto de trabalhos oriundos do Plano Nacional de formação de professores para identificar os principais gargalos quanto à gestão dos resíduos e do lixo na cidade de Alenquer-PA. Os resultados apontam que os problemas da má gestão dos resíduos e do lixo figuram como um reflexo dos problemas políticos enfrentados pela gestão municipal. Os problemas estão atrelados à disputa de poder entre os grupos políticos da cidade que levam à inação política e degradação dos diferentes serviços ofertados à população. Constata-se que os contextos locais e as capacidades têm o poder de limitar os serviços basicos, impulsionar os problemas políticos, sociais e ambientais que colocam a saúde humana em risco.

Palavras-chave: Saúde pública; Gestão municipal; Implementação; Degradação socioambiental.
\end{abstract}

\begin{abstract}
Medical care can prolong life, but health depends on the conditions that cause disease and solid waste and garbage are sources of several health problems. The absence of a more active posture by the State can increase health problems arising from poor management of solid waste and garbage. Studies have indicated problems in waste management in a city in the Amazon context. In this sense, the objective of this article is to identify the causes of the problems of poor management of solid waste and garbage in this city in the Amazon context from the analysis of these works. To this end, a documentary analysis of a set of works from the National Plan for teacher training was used to identify the main bottlenecks regarding waste and garbage management in the city of Alenquer-PA. The results show that the problems of poor waste and garbage management are only a reflection of the political problems faced by municipal management. The problems are linked to the power struggle between political groups in the city that lead to political inaction and degradation of the different services offered to the population. It appears that local contexts and capacities have the power to limit basic services, to boost political, social and environmental problems that put human health at risk.
\end{abstract}

Keywords: Public health; Municipal management; Implementation; Socio-environmental degradation.

\section{Resumen}

La atención médica puede prolongar la vida, pero la salud depende de las condiciones que causan enfermedades y los desechos sólidos y la basura son fuentes de varios problemas de salud. La ausencia de una postura más activa por parte del Estado puede agravar los problemas de salud derivados del mal manejo de los residuos sólidos y la basura. 
Los estudios han indicado problemas en la gestión de residuos en una ciudad en el contexto de la Amazonía. En este sentido, el objetivo de este artículo es identificar las causas de los problemas del mal manejo de los residuos sólidos y la basura en esta ciudad del contexto amazónico a partir del análisis de estos trabajos. Para ello, se utilizó un análisis documental de un conjunto de trabajos del Plan Nacional de Formación Docente para identificar los principales cuellos de botella en la gestión de residuos y basuras en la ciudad de Alenquer-PA. Los resultados muestran que los problemas de mala gestión de residuos y basuras son solo un reflejo de los problemas políticos que enfrenta la gestión municipal. Los problemas están vinculados a la pugna de poder entre los grupos políticos de la ciudad que conducen a la inacción política y la degradación de los diferentes servicios que se ofrecen a la población. Parece que los contextos y las capacidades locales tienen el poder de limitar los servicios básicos, de potenciar los problemas políticos, sociales y ambientales que ponen en riesgo la salud humana.

Palabras clave: Salud pública; Gestión municipal; Implementación; Degradación socioambiental.

\section{Introduçãa}

Os cuidados médicos podem prolongar a vida e melhorar o prognóstico após algumas doenças graves, porém o mais importante para a saúde da população são as condições sociais e econômicas que tornam as pessoas doentes (Wilkinson \& Marmot, 2003). Assim, pessoas só se encontram seguras quando há recursos que inibem ou diminuem as situações de riscos à saúde.

Apesar disso, quando pensamos em segurança humana não devemos nos preocupar, unicamente, com eventos catastróficos provocados por algum tipo "arma", mas no conjunto de condicionantes que colocam a vida humana em risco (King \& Murray, 2001). Muitos eventos que colocam a vida humana em risco estão ligados às questões que refletem a vulnerabilidade da vida cotidiana. Portanto, a segurança humana está diretamente correlacionada com as condições do ambiente em que vivem.

Um dos riscos cotidianos que exerce efeitos diretos sobre os seres vivos e o ambiente é o lixo. Com a industrialização, a produção de lixo no mundo teve um aumento significativo. O lixo descartado inadequadamente acaba se tornando abrigo para transmissores de doenças, como insetos e roedores responsáveis por doenças como febre tifoide, cólera, amebíase, disenteria, giardíase, ascaridíase, leishmaniose, febre amarela, dengue, malária, leptospirose e peste bubônica (Barbosa \& Barbosa, 2014). Os impactos negativos ao meio ambiente variam desde a contaminação de corpos d'água e do solo à poluição do ar e do visual entre outros tipos de contaminação ambiental.

Com o aumento da produção de lixo, ocorre a liberação de gases que provocam o efeito estufa, isto é, um problema ambiental que impacta às gerações futuras. No Brasil, cerca de cerca de 75 milhões de pessoas no Brasil, ou 1/3 da população, sofrem com os males da gestão inadequada de resíduos sólidos (Greenme, 2015). Este problema é mais recorrente em municípios menores.

Nos municípios, cabe ao governo municipal assegurar as condições de saneamento ambiental para garantir a saúde da comunidade. Entretanto, em muitos municípios as organizações públicas apresentam uma visão limitada acerca dos problemas oriundos da má gestão dos resíduos sólidos (Guerrero; Maas \& Hogland, 2013; Matheus; Freitas; Oliveira; Okawara; Silva \& Silva, 2019). Na maioria das vezes, a falta capacidades impede a adoção de uma série de medidas que gerariam ganhos tanto para a sociedade quanto para o meio ambiente de um modo geral (Guerrero et al., 2013; Souza, Boeira, Silva, \& Junkes, 2008; Yang, Chen, Liu, Gong, Yu \& Wang, 2013) e o resultado se faz presente em todo processo de saneamento e gestão ambiental.

Entende-se por capacidades o conjunto de recursos e habilidades usados pelas organizações públicas para monitorar o ambiente, mediar interesses e implementar soluções políticas que maximizam os objetivos coletivos (Mann, 1984; Wu, Ramesh, Howlett, 2015). É por meio do conjunto de habilidades e recursos que as organizações fazem frente aos grupos de interesses da sociedade. No entanto, em sociedades nas quais as organizações públicas enfrentam grupos organizados e poderosos, elas acabam sendo capturadas e passam a legitimar os interesses privados (Migdal, 1988). Consequentemente, os interesses destes grupos inibem o governo de assumir uma postura mais ativa no que tange ao saneamento e gestão ambiental 
adequados criando uma pluralidade de eventos que, interdependentemente, podem afetar a integridade física, biológica e psíquica do ser humano.

Grande parte destes problemas são oriundos da capacidade de gestão dos municípios (Deus, Battistelle \& Silva, 2015; Garcia, Costa, Colares, Sousa, \& Santos, 2019; Guerrero et al., 2013; Yang et al., 2013), do poder econômico do município, uma vez que a gestão dos resíduos e do lixo demanda de 20 a $40 \%$ do orçamento. Logo, o gestor passa a ter que fazer escolhas ou trata os resíduos e lixo ou oferta outros bens e serviços à sociedade (Matheus et al., 2019; Yang, Chen, Liu, Gong, Yu, \& Wang, 2013). Os municípios com menor capacidade financeira para gerir resíduos e o lixo estão mais propensos ao aparecimento de inúmeras doenças, bem como prevalece a falta de atenção à saúde (Barros, César, Carandina, \& Torre, 2006; Colon-Gonzalez, El Rayess, Guevara, \& Anandarajah, 2015), dificuldade no acesso aos serviços hospitalares (Obrist, Iteba, Lengeler, Makemba, Mshana, Nathan, Alba, Dillip, Hetzel, Mayumana, Schulze \& Mshinda, 2007), más condições infraestruturais e socioeconomicos (Mann, 1984), a distância e falta de recursos financeiros (Nemet \& Bailey, 2000), o baixo nivel de educação (Silva \& Teixeira, 2016) e outros condicionantes expoem as pessoas a maior vulnerabilidade cotidiana.

De forma objetiva, estes elementos estão ligados aos determinantes sociais da saúde e da doença (Kaufman et al., 2017; Wilkinson \& Marmot, 2003), que afetam as oportunidades das pessoas de levarem vidas saudáveis. São os condicionantes que configuram o atual estado da arte da segurança humana (Programa das Nações Unidas para o Desenvolvimento [PNUD], 1994), ou seja, saindo de uma visão unicamente da segurança física para uma visão ampliada voltada para a vida humana. Neste sentido, pesquisas de conclusão de curso do Plano Nacional de Formação de Professores da Educação Básica (PARFOR) da Universidade Federal do Oeste do Pará (UFOPA) têm apontado problemas quanto à gestão dos resíduos sólidos na cidade de Alenquer-PA. Em razão disso, questiona-se quais as relações que se podem extrair dos problemas da gestão do lixo em Alenquer com a capacidade das organizações públicas responsáveis pela gestão dos Resíduos e do lixo? Quais as relações que se pode fazer dos problemas da gestão do lixo e a saúde em Alenquer?

Os problemas são causados por um conjunto de fatores inter-relacionados provocando um fluxo circular de causa e efeito que se propaga no tempo. Para saná-los, exige-se uma abordagem holística abrangente que oferte caminhos para melhorias duradouras (Bachrach \& Baratz, 2011). Fato que torna relevante investigar as origens das deficiências na gestão dos resíduos sólidos e do lixo na cidade de Alenquer-PA. Para tanto, o objetivo deste artigo é identificar quais são as causas dos problemas da má gestão dos resíduos sólidos e do lixo em Alenquer segundo os estudos do PAFOR.

Espera-se que este artigo possa refletir a realidade de muitas cidades brasileira, bem como servir de alerta para as autoridades públicas repensarem a gestão do lixo nos planos políticos e estratégicos locais, no sentido de não apenas atender as mínimas condições de saúde, como também ampliar a segurança humana para poupar recursos escassos que, caso sejam poupados com as melhorias nas condições de saúde, poderão ser investidos em outras áreas de interesse social e/ou coletivos da sociedade. Além dessa parte introdutória, o artigo contempla mais três partes. A segunda traz o delineamento da pesquisa. Na terceira seção, há uma discussão sobre a problemática da gestão do lixo em Alenquer, por fim, as considerações finais.

\section{Metodologia}

Tendo por base os objetivos, este estudo apresenta caráter exploratório, uma vez que estudos acerca da gestão do lixo no contexto amazônico, especificamente em Alenquer-PA, são escassos, logo o estudo tem o intuído de proporcionar maior familiaridade com o problema em estudo (Gil, 2002; Lakatos \& Marconi, 2010). A vista disso, optou-se por uma abordagem qualitativa para explorar informações mais subjetivas e aprofundadas por meio de uma pesquisa bibliográfica, pois permite ao pesquisador se familiarizar e melhor compreender o tema em estudo (Creswell, 2010; Godoy, 1995). A bibliografia base do estudo foram trabalhos de conclusão de curso do PARFOR do Campus Universitário de Alenquer (CALE), que abordaram a problemática do lixo, da saúde e da gestão municipal como temáticas principais. 
Os trabalhos foram levantados por meio da Biblioteca do CALE e foram tendo como descritores de buscas os seguintes vocábulos: "lixo", "serviços públicos", "gestão pública” "políticas públicas", "infraestrutura" e "impactos ambientais" nos títulos, resumos e palavras-chaves. Nesse sentido, foram selecionados 6 (trabalhos) que, juntos, formaram o corpus analítico do problema abordado. Em seguida, o corpus foi analisado com base nas técnicas de análise de conteúdo descritas por Bardin, (1977), por ser a obra de referência no campo da análise de conteúdo (Camargo, 2005; Ratinaud, 2009).

Os trabalhos foram analisados no software de mapeamento estatístico IRAMUTEQ. O software identifica a estrutura de correlação e inter-relação dos vocábulos em um texto (Marchand \& Ratinaud, 2012). Após a estrutura de correlação, as palavras são distribuídas em classes explicativas que estão hierarquicamente distribuídas formando uma estrutura do contexto geral para o específico, processo similar à análise de conteúdo.

Para tanto, o material foi preparado de acordo com as especificações do software. Cada trabalho foi identificado por uma linha de comando e as variáveis analisadas. A linha de comando é formada por 4 (quatro) asteriscos seguidos pela identificação do trabalho logo após a identificação das variáveis analisadas, como se pode observar na Figura 1:

Figura 1. Linha de comando para análise do corpus no Iramuteq.

*****TCC_1 *ANO_01 *GESTÃO_PÚBLICA *LIXO.....

Fonte: Autores.

O método analítico foi a Análise Fatorial de Correspondência (AFC) e Análise de Similitude. Tais métodos permitem estudar as relações entre os objetos de um determinado conjunto de modo que favorece o agrupamento e classificação dos vocábulos acordo com o grau de associação. Logo, de acordo com a frequência de coocorrência da palavra no texto é possível classificá-los em grupos explicativos interligados formando conexões que se assemelham a uma árvore (Fallery \& Rodhain, 2007; Marchand \& Ratinaud, 2012), para fundamentar as discussões que se seguem.

\section{Resultados e Discussão}

O corpus analisado era formando por 978 segmentos de textos com 33846 palavras, das quais 9262 eram substantivos, 3134 eram adjetivos, 2859 verbos e 1562 advérbios, ou seja, palavras que carregam significados e denotam ação. No geral, lixo é o vocábulo que mais aparece. Esse conjunto de vocábulos oferece uma visão ampla dos condicionantes que produzem a deterioração do tecido econômico e social no município. A partir destes vocábulos se tem uma visão descritiva de constructos sociodemográficos e políticos que ajudam a entender a problemática estudada. Portanto, tais palavras permitiram estudar a correlação entre a problemático do lixo e a gestão pública municipal no sentido de descrever a vulnerabilidade humana do município.

O conjunto de palavras se agrupa em 5 clusters explicativos. O primeiro cluster é denominado elementos da gestão pública. São vocábulos que rementem ao gestor público, aos recursos, à agenda, à implementação de políticas públicas entre outras. O segundo clusters é um reflexo da ação do gestor público, remente aos serviços públicos ofertados à sociedade, tais como saúde, educação, lazer, cultura entre outros. Esse cluster foi denominado serviços públicos. O terceiro clusters é denominado tipos de resíduos. Compõem esse agrupamento de palavras elementos como vidro, plástico, matéria orgânica, entre outros. O quarto agrupamento de palavras, denominado riscos ambientais e sociais locais, engloba elementos como contaminação, doenças, descarte, impacto, entre outros. Por fim, a aglomeração denominada local. Esse cluster traz a relação espacial dos problemas enfrentados pela população alenquerense. Compõe esse grupo palavras como bairro, rua, escola etc., como se observa na Figura 2. 
Figura 2. Correlação explicativa entre os vocábulos no corpus.

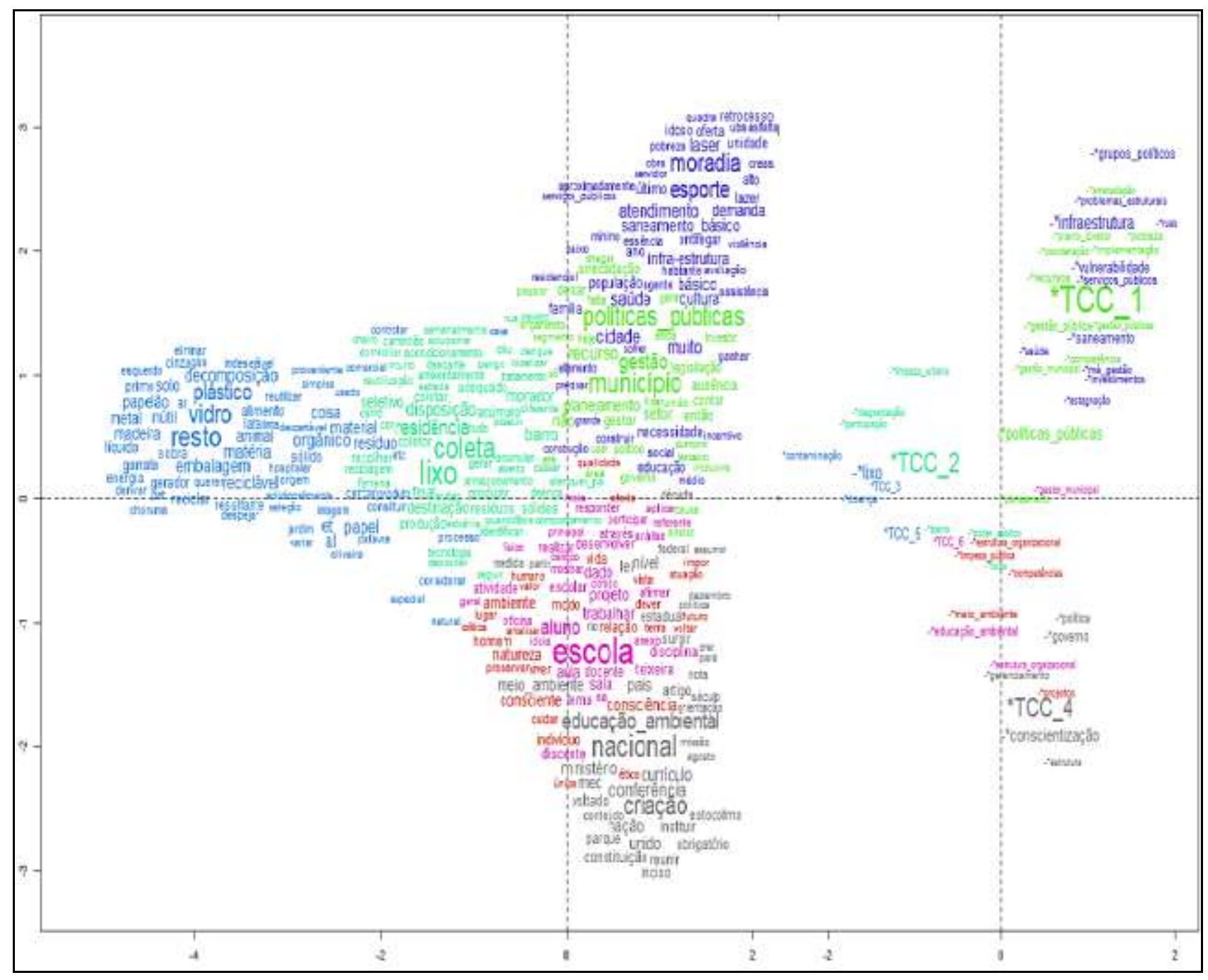

Fonte: Elaborado a partir do resultado da Análise Fatorial de Correspondência do Iramuteq.

As palavras que formam os clusters se inter-relacionam com as variáveis explicativas. Logo, elementos como plano diretor, plano municipal, infraestrutura, lixo, gestão municipal entre outras estão diretamente correlacionados com as variáveis estagnação, má gestão, infraestrutura, descaso, prestação de contas entre outras, bem como os problemas relatados nos bairros. Esses problemas são oriundos problemas na gestão pública, evidenciados por varáveis como planejamento, recursos, governos, políticas públicas e dos grupos políticos.

Tais relações de conexidade formam a árvore de inter-relação que tem na localidade, Alenquer, o cerne da problemática. Nesse sentido, a localidade é o centro de discussão e as linhas mais espeças representam maior conexidade com os problemas apresentados nesse espaço geográfico. Portanto, pela Figura 3, observa-se a conexão do local com o público, com as políticas públicas, com os aspectos sociais e, sobretudo, com o papel do político. 
Figura 3. Centralidade de alguns constructos na explicação da problemática

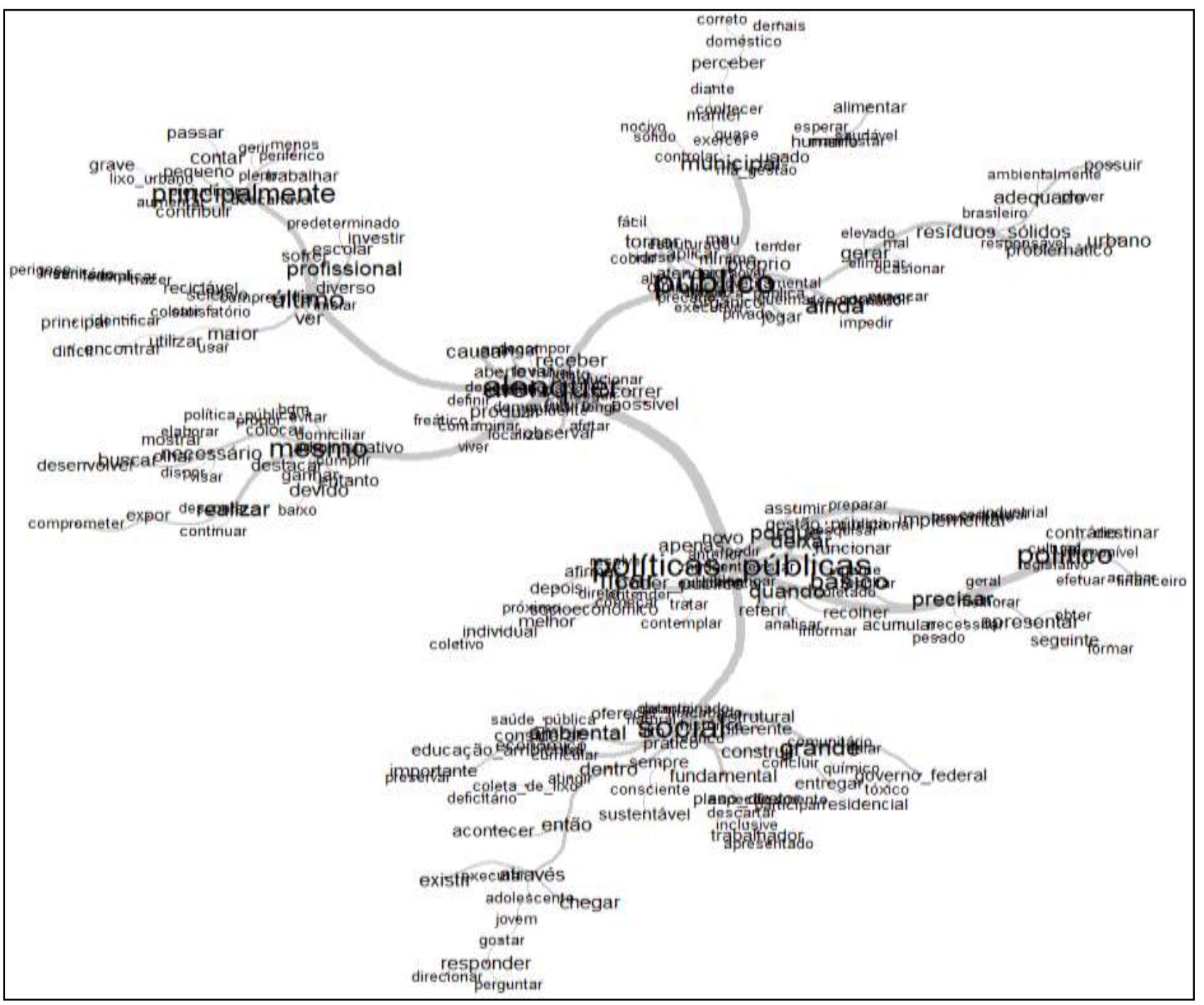

Fonte: Resultado da Análise de Similitude do Iramuteq.

A ação do governo pode sofrer influências no desenho das políticas públicas, na implementação e na avaliação, pois o jogo político é um campo de disputas de poder, no qual a persuasão, a barganha e/ou a negociação figuram como fatores que definem o que é realizável (Bachrach \& Baratz, 2011; Souza, 2012). Nesse sentido, o foco analítico destina-se aos participantes dos processos, visto que as políticas públicas são pensadas por pessoas para resolver os problemas de outras pessoas. Assim, o foco da problemática se volta para os gestores públicos e, sobretudo, para os grupos políticos.

Os grupos políticos podem trabalhar para expandir o debate sobre os problemas locais ou buscam restringir o debate criando ponto de veto. A luta política, muitas vezes, acaba por limitar a efetividades das deliberações sob políticas públicas. Os atores orientam o jogo político buscando estabelecer políticas que favoreçam interesses individuais.

Esses jogos de interesses vão atribuindo poder a determinados grupos, de tal modo que estes podem manipular os valores sociais e políticos (mobilização viés) para alcançar seus interesses ou limitam interesses julgados impróprios, configurando uma agenda reativa ou proativa (Souza, 2012). Desta forma, os grupos poderosos tentam manter o monopólio das questões que integram a agenda pública, fato que tona as mudanças lentas. Logo, na cidade de Alenquer há uma perpetuação de grupos políticos e oligarquias que decidem quais políticas de conveniências estão adequados aos seus interesses (Santos, 2017). 
Com isso, a agenda política local é dominada por grupos que, organizados em redes, têm a capacidade de dominar a tomada de decisão, fazendo valer seus interesses. Neste contexto, as decisões políticas são tomadas com base no clientelismo político, de tal forma que há descaso com os serviços públicos, fato que limita a atenção à saúde (Barros et al., 2006; ColonGonzalez et al., 2015), torna precário os serviços hospitalares (Obrist et al., 2007), entre outros elementos indutores de vulnerabilidade cotidiana aos cidadão da cidade.

De modo objetivo, os recursos escassos da sociedade, que poderiam ser empregados em programas educacionais, na saúde, segurança, direitos civis etc., acabam não produzindo efeitos benéficos à população. Consequentemente, esse processo se faz mais presente onde as instituições são frágeis, onde há pouca capacidade de regulação e responsabilização dos envolvidos nos subsistemas de governo (Guerrero et al., 2013; Yang et al., 2013). Essa realidade é descrita pela relação entre as variáveis descritivas dos tipos de resíduos com os riscos ambiental e sociais locais. Elementos como coleta de lixo, saúde pública, doença e queima estão diretamente ligados à variável Alenquer. Tal relação leva a perda de foco de implementação das políticas públicas pelo setor político da cidade de Alenquer (Rosário, 2016; Santos, 2017; Sousa \& Aquino, 2017), o que tem causado desperdício de recursos públicos evidenciando a falta de vontade política e o descaso com os problemas locais.

A falta de vontade políticas é um reflexo da ação dos grupos políticos que lutam pelo poder tirando o foco do poder executivo dos serviços e orientando-o para o poder político (Migdal, 1988; Santos, 2017). Dessa forma, as ações não são implementadas ficando a cargo de outras gestões que têm outras prioridades. Portanto, o que foi planejado é ignorado. Como relatam Santos (2017), Silva e Garcia (2017), a continuidade das políticas não tem sido efetuada pelas gestões, pelo contrário, tem sido atropelada em todo seu curso pelas circunstâncias políticas municipais que não têm o planejamento urbano como prioridade. A inação política se reflete na precariedade dos serviços ofertadas à população. Para citar a incapacidade ética dos políticos, que usam de má índole para chegar ao poder, limitam a efetividade do uso dos recursos. Como o município é dependente do fundo de participação municipal para implementação e manutenção das políticas públicas, há uma inter-relação entre as condições do município com as disponibilidades de recursos com a gestão e, sobretudo, com a ação dos políticos locais, como se pode observar na Figura 4. 
Figura 4. Interdependência explicativa entre os constructos recursos e município.

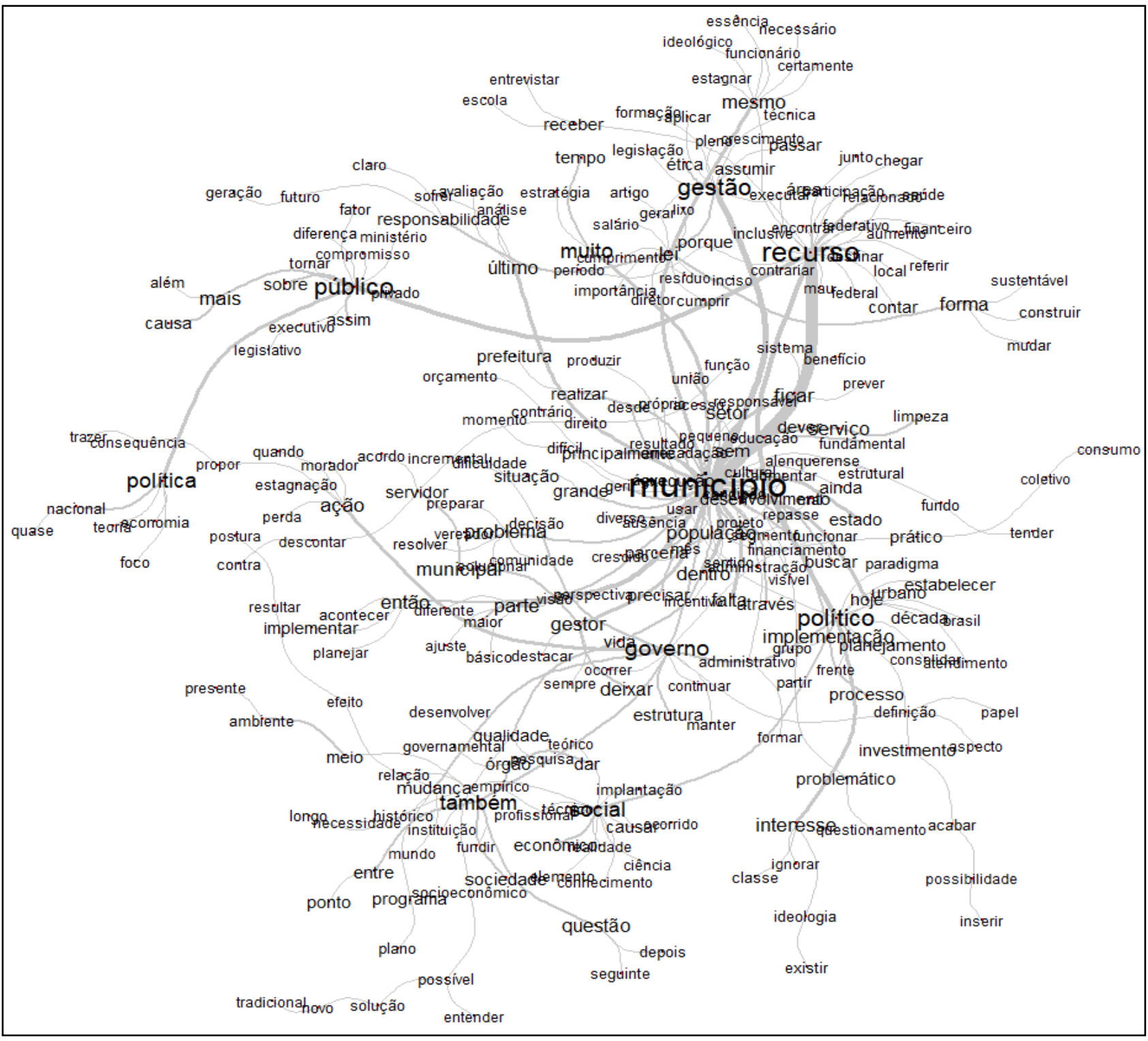

Fonte: Resultado da Análise de Similitude do Iramuteq

A inter-relação entre localidade, recursos, agentes políticos e a falta de ética dos gestores coloca o planejamento e a implementação de políticas públicas em segundo plano. O reflexo é na infraestrutura e nas condições sociais da cidade. Assim, há uma falta de zelo e presteza aos recursos públicos que são usados indevidamente de maneira aleatória e irresponsável resultando em obras inacabadas abandonadas e mal-estruturadas (Santos, 2017). Ainda segundo o autor, a falta de transparência das prestações de conta da administração pública ainda é um problema e a câmara municipal não exerce seu papel com imparcialidade.

Esse conjunto de gargalos observados compromete a efetivação das necessidades sociais em uma cidade que foi crescendo desordenadamente. Os bairros não foram planejamentos, eles foram surgindo aleatoriamente, então, os problemas se tornaram crescentes, sendo o recolhimento do lixo um dos serviços públicos mais deficitários (Chaves \& Oliveira, 2016; Silva \& Garcia, 2017; Sousa \& Aquino, 2017). Em alguns bairros, as ruas são asfaltadas enquanto outras são de chão batido, em especial os bairros periféricos.

A falta de infraestrutura dos bairros periféricos dificulta o tráfego dos pedestres e dos veículos coletores de lixos e ambulâncias. Outro problema e a falta de lixeira nas ruas da cidade, fazendo com que os habitantes joguem lixo em vias 
públicas, o que não ocorre em bairros com melhor estrutura (Chaves \& Oliveira, 2016). Nesse sentido, há uma relação de conexidade entre lixo e bairro. Além da coleta ser ineficiente, é orientada para grupos sociais privilegiados. (Figura 5).

Figura 5. Correlação explicativa entre os constructos lixo e bairro no município de Alenquer.

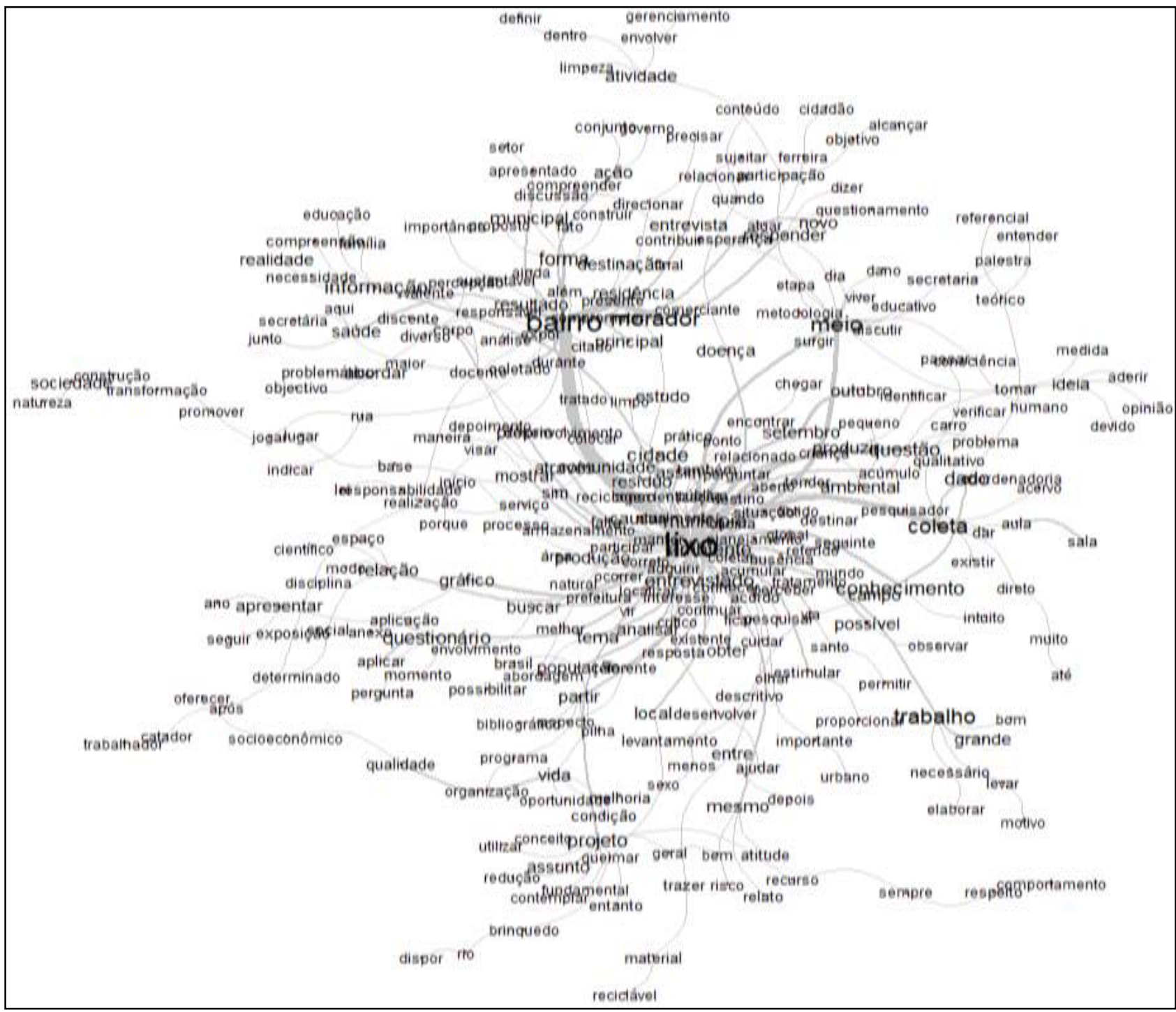

Fonte: Resultado da análise de similitude do Iramuteq.

O lixo é apenas um reflexo do aumento dos problemas públicos que ampliam os problemas sociais, em especial ameaçam a saúde pública e o ambiente na cidade de Alenquer-PA. A inefetividade dos serviços de coleta de lixo aliada com os hábitos domésticos tem aumentado a incidência de doenças como leishmaniose, visceral e tegumentar a visceral, aumento do número de casos de dengue e a presença de um índice alto de casos de malária (Chaves \& Oliveira, 2016; Sousa \& Aquino, 2017).

No geral, as implicações evidenciam os problemas na gestão pública da cidade de Alenquer e limitam a identificação dos problemas sociais que assolam um Estado, bem como tomam medidas paliativas como projetos de coleta seletiva, reciclagem e na área de educação ambiental, visto que a população é carente de informações em relação à problemática do lixo e o seu impacto no meio ambiente (Fu et al., 2013; Rosário, 2016). A inação política inibe a construção de uma sociedade 
capaz de ser atora do próprio desenvolvimento. Para tanto, essa sociedade precisa se organizada para ter poder nas deliberações junto aos grupos que dominam a agenda política.

A necessidade de organização social se faz necessário uma vez que o interesses de grupos mais organizados são superiores aos interesses sociais, fato que torna o plano plurianual do município um documento inútil, pois fica somente no papel o planejamento das políticas públicas (Chaves \& Oliveira, 2016; Santos, 2017), refletindo mais o jogo de interesses na busca pelo poder que uma organização político-administrativo eficiente e eficaz (Bachrach \& Baratz, 2011; Guerrero et al., 2013; Mann, 1984; Wu et al., 2015). Desta forma, os gargalos enfrentados pela sociedade alenquerense são reflexos da ineficiência políticas em diferentes frentes, não somente na gestão dos resíduos sólidos, são reflexos da falta de infraestrutura, da precariedade dos serviços e das políticas que refletem no desenvolvimento social e ambiental da cidade.

A causa direta desses problemas são as disputas políticas entre os grupos políticos. Esses grupos dominam a agenda política orientados, unicamente, em alcançar o poder acabam por ampliar os níveis de insegurança humana na cidade. Assim, as inter-relações pessoais ganham destaque nos estudos sobre as condições políticas, econômicas, sociais e de saúde em Alenquer. Neste sentido, as redes, mas, sobretudo, o papel de determinados atores desempenham função impar no pensar e implementar as políticas públicas locais.

A gestão pública não deve se diferenciar da gestão de qualquer outra atividade tendo em vista que o estudo da implementação é o estudo do comportamento organizado em uma estrutura de poder hierarquizada (Wu et al., 2015). As mudanças nesse sistema político serão lentas, pois há indícios de não exista vontade política das elites locais.

\section{Conclusão}

Muitos dos problemas de saúde pública estão relacionados à gestão dos resíduos sólidos e do lixo. Entretanto, muitos municípios encontram dificuldades para gerir eficientemente seus resíduos sólidos e o lixo. As principais deficiências estão atreladas aos fatores econômicos e a capacidade de gestão. Em razão disso, este artigo buscou identificar os problemas da má gestão dos resíduos e do lixo em uma cidade no contexto amazônico.

Canstatou-se que o problema do lixo é apenas uma das consequências da ação dos grupos políticos que atuam na cidade. Esses grupos interessados no poder monopolizam a agenda política e relegam os problemas sociais e insfraestruturais a segundo plano. Nesse contexto, os serviços públicos são precários em áreas como infraestrutura das ruas, da saúde, da educação, do esporte, do lazer, da cultura, bem como nos serviços de coleta de lixo.

A causa imediata desses problemas transcende o planejamento, pois a busca pelo poder limita vontade e interesse dos grupos políticos. Destarte, o sucesso do pensar as políticas e implementá-las depende das características de quem controla o processo e como se exercita este controle pode refletir no níveis de desenvolvimento local. Constata-se que os contextos locais e as capacidades têm o poder de limitar os serviços básicos, impulsionar os problemas políticos, sociais e ambientais que colocam a saúde humana em risco.

Apesar disso, novos estudos precisam ser realizados para conhecer mais detalhadamente as características dos burocratas que planejam e implementam as políticas públicas, se há problemas intraorganizacionais impactando na coordenação das ações públicas, bem como demanda-se estudos comparativos com outros municípios circunvizinhos para se identificar se é apenas um problema local ou se é um problema recorrente nas gestões da regionalidade.

\section{Referências}

Bachrach, P., \& Baratz, M. S. (2011). Duas faces do poder. Revista de Sociologia e Política, 19(40), 149. http://search.proquest.com/openview/00832cad960ed4e418187070f4d254f5/1?pq-origsite=gscholar 
Bardin, L. (1977). Análise de Conteúdo (70th ed.). Persona.

Barros, M. B. de A., César, C. L. G., Carandina, L., \& Torre, G. D. (2006). Desigualdades sociais na prevalência de doenças crônicas no Brasil, PNAD-2003. Ciência \& Saúde Coletiva, 11(4), 911-926. http://www.scielo.br/scielo.php?script=sci_arttext\&pid=S1413-81232006000400014\&lng=pt\&nrm=iso\&tlng=pt

Camargo, B. V. (2005). ALCESTE: um programa informático de análise quantitativa de dados textuais. In A. S. P. Moreira (Ed.), Perspectivas teóricometodológicas em representações sociais. (pp. 511-539). UFPB/ Universitária.

Chaves, E. D. S., \& Oliveira, E. N. (2016). A produção e coleta do lixo urbano no bairro aningal em Alenquer-PA. Trabalho de Conclusão de Curso, 25 p. Alenquer-PA. Universidade Federal do Oeste do Pará.

Colon-Gonzalez, M. C., El Rayess, F., Guevara, S., \& Anandarajah, G. (2015). Successes, challenges and needs regarding rural health medical education in continental Central America: a literature review and narrative synthesis. Rural \& Remote Health, 15(3), 3361.

Creswell, J. W. (2010). Projeto de Pesquisa: Métodos Qualitativos, Quantitativos e Mistos. .pArtmed.

Deus, R. M., Battistelle, R. A. G., \& Silva, G. H. R. (2015). Resíduos sólidos no Brasil: contexto, lacunas e tendências. Engenharia Sanitaria e Ambiental, 20(4), 685-698. https://doi.org/10.1590/S1413-41522015020040129347

Fallery, B., \& Rodhain, F. (2007). Quatre approches pour l'analyse de données textuelles. XVIe Conférence AIMS, 28(3), 3-17. https://hal.archivesouvertes.fr/hal-00821448

Fu, H.-Z., Wang, M.-H., \& Ho, Y.-S. (2013). Mapping of drinking water research: A bibliometric analysis of research output during 1992-2011. Science of The Total Environment, 443, 757-765. https://doi.org/10.1016/j.scitotenv.2012.11.061

Garcia, M. C. L., Costa, M. A. de J., Colares, J. A. da C., Sousa, H. A. de, \& Santos, M. A. O. (2019). Organizational disconnection and its impacts on the effectiveness of public cleaning in the municipality of Alenquer-PA. ITEGAM-Journal of Engineering and Technology for Industrial Applications (ITEGAMJETIA), 5(18). https://doi.org/10.5935/2447-0228.20190027

Gil, A. (2002). Como elaborar projetos de pesquisa. In São Paulo (5th ed.). Atlas.

Godoy, A. S. (1995, June). Pesquisa qualitativa: tipos fundamentais. Revista de Administração de Empresas, São Paulo, 35(3), 20-29. https://doi.org/10.1590/S0034-75901995000300004

Greenme. (2015). Má gestão do lixo causa problemas de saúde à toda sociedade. https://www.greenme.com.br/informar-se/lixo-e-reciclagem/2421-magestao-do-lixo-causa-problemas-de-saude-a-toda-sociedade

Guerrero, L. A., Maas, G., \& Hogland, W. (2013). Solid waste management challenges for cities in developing countries. Waste Management, 33(1), 220-232. https://doi.org/10.1016/j.wasman.2012.09.008

Kaufman, A., Rhyne, R. L., Anastasoff, J., Ronquillo, F., Nixon, M., Mishra, S., Poola, C., Page-Reeves, J., Nkouaga, C., Cordova, C., \& Larson, R. S. (2017). Health Extension and Clinical and Translational Science: An Innovative Strategy for Community Engagement. The Journal of the American Board of Family Medicine, 30(1), 94-99. https://doi.org/10.3122/jabfm.2017.01.160119

King, G., \& Murray, C. J. (2001). Rethinking human security. Political Science Quarterly, 116(4), 585-610.

Lakatos, E. M., \& Marconi, M. D. A. M. (2010). Fundamentos de metodologia científica (7th ed.). Atlas.

Mann, M. (1984). The autonomous power of the state: its origins, mechanisms and results. European Journal of Sociology, 25(02), 185. https://doi.org/10.1017/S0003975600004239

Marchand, P., \& Ratinaud, P. (2012). L'analyse de similitude appliqueé aux corpus textueles: les primaires socialistes pour l'election présidentielle française. Actes Des 11eme Journées Internationales d'Analyse Statistique Des Données Textuelles. JADT, 687-699.

Matheus, M., Freitas, J. A. de, Oliveira, A., Okawara, J. M., Silva, L. M. da, \& Silva, A. (Eds.). (2019). Índice De Sustentabilidade Da Limpeza Urbana (4th ed., Vol. 4, Issue 3). Selur.

Migdal, J. S. (1988). Strong societies and weak states : state-society relations and state capabilities in the Third World. Princeton University Press.

Nemet, G. F., \& Bailey, A. J. (2000). Distance and health care utilization among the rural elderly. Social Science \& Medicine, 50(9), 1197-1208. https://doi.org/10.1016/S0277-9536(99)00365-2

Obrist, B., Iteba, N., Lengeler, C., Makemba, A., Mshana, C., Nathan, R., Alba, S., Dillip, A., Hetzel, M. W., Mayumana, I., Schulze, A., \& Mshinda, H (2007). Access to Health Care in Contexts of Livelihood Insecurity: A Framework for Analysis and Action. PLoS Medicine, 4(10), e308. https://doi.org/10.1371/journal.pmed.0040308

PNUD. Programa das Nações Unidas para o Desenvolvimento (1994). Human Development Report. Oxford University Press.

Ratinaud, P. (2009). IRAMUTEQ: Interface de R pour les Analyses Multidimensionnelles de Textes et de Questionnaires. http://www.iramuteq.org/frontpage/presentation_view

Rosário, E. M. F. Do. (2016). Abordagem da educação ambiental: lixo e reciclagem nas escolas no município de Alenquer-PA. Trabalho de Conclusão de Curso, 42 p. Alenquer. Universidade Federal do Oeste do Pará.

Santos, G. F. R. Dos. (2017). A ausênica de políticas públicas no município de Alenquer: como romper o paradigma da estagnação que impede o crescimento socioeconomico nos últimos 8 anos. Trabalho de Conclusão de Curso, 35 p. Alenquer-PA. Universidade Federal do Oeste do Pará. 
Research, Society and Development, v. 10, n. 5, e54210515221, 2021

(CC BY 4.0) | ISSN 2525-3409 | DOI: http://dx.doi.org/10.33448/rsd-v10i5.15221

Silva, A. pereira da, \& Garcia, J. da silva. (2017). O tratamento do lixo na escola municipal de educação infantil e ensino fundamental Izolina d'Assunção Lopes Valente, município de Alenquer-PA. Trabalho de Conclusão de Curso, 30p. Alenquer-PA. Universidade Federal do Oeste do Pará.

Silva, H. S. da, \& Teixeira, M. G. C. (2016). A influênica doa ators na institucionalização do meio ambiente e do desenvolvimento regional em uma instituição federal de educação. Revista Brasileira de Gestão e Desenvolvimento Regional, 12(3). http://www.rbgdr.net/revista/index.php/rbgdr/article/view/2525/544

Sousa, E. S, \& Aquino, F. S. (2017). O risco de contaminação no trabalho em lixões no município de Alenquer-PA. Trabalho de Conclusão de Curso, 23 p. Alenquer-PA. Universidade Federal do Oeste do Pará.

Souza, C. (2012). Políticas Públicas: Conceitos, Tipologias e Sub-Áreas *. Fundação Luís Eduardo Magalhães, 1-29. https://xa.yimg.com/kq/groups/22690463/755780646/name/políticas+públicas+conceitos+tipologias+e+subáreas.pdf

Souza, M. V. De, Boeira, S. L., Silva, W. V. K. M., \& Junkes, R. V. (2008). Políticas Públicas de Gerenciamento do Lixo Urbano no Brasil. Universidade Do Vale de Itajai. http://base.d-p-h.info/pt/fiches/dph/fiche-dph-7834.html

Wilkinson, R., \& Marmot, M. (2003). Social Determinants of Health: the Solid Facts. World Health Organization, 2(2), 1-33. https://doi.org/10.1016/j.jana.2012.03.001

Wu, X., Ramesh, M., \& Howlett, M. (2015). Policy capacity: A conceptual framework for understanding policy competences and capabilities. Policy and Society, 34(3-4), 165-171. https://doi.org/10.1016/j.polsoc.2015.09.001

Yang, L., Chen, Z., Liu, T., Gong, Z., Yu, Y., \& Wang, J. (2013). Global trends of solid waste research from 1997 to 2011 by using bibliometric analysis. Scientometrics, 96(1), 133-146. https://doi.org/10.1007/s11192-012-0911-6 